levipes, and the reclusive $R$. niobe below $M$. rubex; these departures are easily rationalised by appeal to differences between genera (for example, each Melomys is larger than its Rattus partner).

Additional support for the idea that the distribution patterns are governed by the amount of disturbance by man and pigs comes from the archaeological record. White's (op. cit.) excavations have shown a marked increase in the representation of small rodents in the upper horizons of his site. Tooth measurements suggest that the three species present are $R$. niobe, $R$. exulans and $R$. ruber; of these, the first is found only in lower levels, the second only in the top level, while the third increases over time in step with increasing signs of human activity.

Dwyer concludes "that the local diversity of small terrestrial rodents in rain forests of densely populated areas of the New Guinea highlands was higher in the past than it is today and that reduction has been a consequence of habitat disturbance by men and pigs producing a smaller, less continuous, area of rainforest and simplification of ground cover. The pattern of local species distributions is now dominated by gross disturbance effects with the more opportunistic of rainforest species found in the most disturbed rainforests. Some species may have dropped out of local faunas." $\mathrm{He}$ also suggests that some aspects of the distribution patterns of the montane avifauna of New Guinea (as extensively studied by Diamond and others) may be associated with recent disturbances rather than with long term 'island equilibrium' effects. In common with many other workers, Dwyer sees disturbance as important in promoting and maintaining species diversity, essentially because it enlarges the number of different roles in the evolutionary play. But severe disturbance, of a kind outside the evolutionary experience of the ecosystem, will make for less diversity.

\section{The asthenosphere as point defect zone}

\section{from Peter J. Smith}

AT depths in the Earth of somewhere between $75 \mathrm{~km}$ and $250 \mathrm{~km}$ there is a layer perhaps some $75-150 \mathrm{~km}$ thick in which seismic velocity is slightly lower, and seismic attenuation is rather higher, than in the regions immediately above and below. Any uncertainty implicit in this statement arises because the depth and thickness of the layer, which may in any case be variable, have not been determined everywhere and because, even where experiments have been carried out, the boundaries of the layer are often diffuse. Nevertheless, there seems little doubt that this 'low velocity layer' exists in some form beneath most of the Earth's surface. Moreover, the layer is now usually equated with the asthenosphere, the semi-mobile zone which allows the rigid tectonic plates of the overlying lithosphere to move.

But accepting the identity of the asthenosphere and the low velocity layer, what is the physical cause of the mobility, the lower velocity and the higher attenuation? The majority answer is partial melting of peridotite. Suggestions about the nature of the cavities in which the melt fraction supposedly resides have included spherical pores, coin-shaped cracks and tubular channels; and estimates of the amount of partial melting accordingly required to account for the seismic observations have ranged from about $6 \%$ to $0.1 \%$. Whatever the cavity shape, the degree of partial melting required is apparently low; and so if the low velocity zone were purely a static phenomenon there would be little to choose between models.

The reality is more complicated, however, for the molten material can evidently flow, which means that in at least some parts of the asthenosphere the melt cavities must be interconnected. And that seems to pose quite a problem. For according to Shaw (Geophys. Res. Lett. 5, 629; 1978), Walker et al. (in press) have investigated the stability under gravity of a melt-solid system with interconnected porosity and have thus shown that for tubular channels, instability will arise if the melt fraction exceeds $0.1 \%$ (which compares with the roughly $1 \%$ required to explain the seismic observations). Above $0.1 \%$ the melt segregates and rises from the solid in less than $10^{7} \mathrm{yr}$. Walker and his colleagues therefore conclude that the low velocity layer is either a temporary phenomenon which just happens to be well developed at the moment or a "dynamic system with a melt fraction above their calculated limit of long term stability through which melt must flow continually at a substantial rate, in fact, at a rate which is probably unrealistically high".

Of course, many assumptions are involved, any one or more of which may turn out to invalidate the Walker model as a reasonable semblance of reality. Nevertheless, at the very least the results are such as to suggest that partial melting should not be accepted

Peter J. Smith is a Reader in the Department of Earth Sciences at the Open University. too readily, as, indeed, some workers have long realised. Guegnen and Mercier (Phys. Earth Planet. Interiors 7, 39; 1973), for example, listed several other possibilities and concluded that the seismic properties of the low velocity layer could in theory be as well explained in terms of viscous grain boundary relaxation or dislocationimpurity interactions.

Unfortunately, there is little experimental data to support either of these particular solid state processes as a cause of the asthenosphere; but Shaw has now added one phenomenon for which there is some empirical (and theoretical) support: namely, the presence of point defects. Shaw himself ( $J$. Phys. Chem. Solids 35, 911; 1974) has shown that in the B1 phase of silver iodide, variation in the concentration of lattice point defects leads to a very rapid decrease in sound velocity with increasing temperature and an equally rapid increase with increasing pressure. The magnitudes of defect-produced velocity changes in materials likely to be important in the Earth (such as silicates and oxides) are far less well known and may in any case be different for different types of defect (Frenkel defects, Schottky defects for example). But Shaw has little difficulty in showing that, taking into account the probable relative temperature and pressure effects in materials and the variation of temperature and pressure in the Earth, a seismic velocity anomaly in the Earth would be expected in the depth range $50-250 \mathrm{~km}$ precisely where it is observed.

Considering the uncertainties involved in Shaw's theoretical development, this agreement is amazing. And it is all the more encouraging in that defects are known to facilitate material transport, thus enhancing flow and leading to a decrease in apparent viscosity. If Shaw is right in his thinking, the presence of point defects offers just as reasonable an explanation of the low velocity layer-asthenosphere as does partial.melting, although to prove the point there will have to be far more experiments on probable Earth materials at the appropriate temperatures and pressures.

\section{DNA polymerase and mutation}

from Bryn Bridges

THE underlying importance of damage to DNA and its mutagenic consequences in the production of cancer and certain heritable diseases becomes steadily more apparent, and it may well extend to such superficially unrelated 